\title{
Effective Field Theory and Unitarity in Vector Boson Scattering
}

\author{
Marco Sekulla ${ }^{* a}$, Wolfgang Kilian ${ }^{b}$, Thorsten Ohl $^{c}$, Jürgen Reuter ${ }^{d}$ \\ a Karlsruhe Institute of Technology (DE) \\ $b$ University of Siegen (DE) \\ c Würzburg University (DE) \\ d DESY Theory Group (DE) \\ E-mail: marco.sekulla@kit.edu, kilian@physik.uni-siegen.de, \\ ohl@physik.uni-wuerzburg.de, juergen.reuter@desy.de
}

\begin{abstract}
Weak vector boson scattering at high energies will be one of the key measurements in current and upcoming LHC runs. It is most sensitive to any new physics associated with electroweak symmetry breaking. However, a conventional EFT analysis will fail at high energies. To address this problem, we present a parameter-free prescription valid for arbitrary perturbative and nonperturbative models: the T-matrix unitarization. We describe its implementation as an asymptotically consistent reference model matched to the low-energy effective theory. We show examples of typical observables of vector-boson scattering at the LHC in our unitarized framework. For many strongly-coupled models like composite Higgs models, dimension-8 operators might be actually the leading operators. In addition to those longitudinal and transversal dimension eight EFT operators, the effects of generic tensor and scalar resonances within simplified models are considered.
\end{abstract}

Fourth Annual Large Hadron Collider Physics

13-18 June 2016

Lund, Sweden

${ }^{*}$ Speaker. 


\section{Motivation}

Run I of the LHC has not only revealed a Standard Model-like Higgs boson [1, 2] together with measuring its mass and some of its properties and couplings, but also established the scattering process of electroweak gauge bosons[3, 4, 5] (VBS) as predicted by the Standard Model (SM). This process gives insights into the nature of the electroweak symmetry breaking (EWSB) sector and further fundamental properties of the Higgs. In the SM, the electroweak breaking sector is described as a weakly interacting theory, where the Higgs boson is vastly suppressing the vector boson scattering process at high center-of-mass energies and the scattering amplitude is dominated by the transversal vector boson scattering.

Without the Higgs the VBS scattering amplitudes $V V \rightarrow V V$, where $V$ is $W^{ \pm}, Z$, would rise with $s / v^{2}$ due to the dominant contribution of scalar Goldstone-boson scattering, which represents the longitudinal degrees of freedom of the vector boson scattering. The electroweak interactions would become strongly interacting in the $\mathrm{TeV}$ range. However, the initial limits on VBS are rather weak and only scales close to the pair-production threshold of $\sim 200 \mathrm{GeV}$ are probed. Run II and III of the LHC and future (high-energy) $e^{+} e^{-}$colliders will improve the accuracy and provide new insights in the origin of EWSB. The delicate cancellation between the EW gauge bosons and the Higgs boson in VBS makes this channel an ideal, yet intricate channel to search for new physics.

The discussion in these proceedings is based on our publications in $[6,7,8,9]$ and is an update of [10].

\section{Effective Field Theory, Perturbative Unitarity and Unitarization}

To study new physics in the VBS process generically, we will use the framework of Effective Field Theories (EFT). A set of higher-dimensional operators extends the SM Lagrangian to quantify deviations from the SM, which originate from some new physics at a high energy scale $\Lambda_{i}$ as

$$
\mathscr{L}=\mathscr{L}_{S M}+\sum_{i} \frac{C_{i}}{\Lambda_{i}^{d-4}} \mathscr{O}_{i}^{d}
$$

Here, $C_{i}$ are the associated Wilson coefficients of the operators. Lacking a possibility to disentangle both parameters, we introduce the ratio coupling $F_{i}=\frac{C_{i}}{\Lambda_{i}^{d-4}}$.

Many different operator bases have been proposed for the electroweak sector, an overview and also translations between them have been discussed e.g. in [11, 12]. For illustrative purposes, we study only the subset of operators which purely contribute to the Higgs sector and therefore affect only the coupling of longitudinal vector bosons and the Higgs boson. This subset of operators contains $\mathscr{O}_{H D}$ as a dim-6 operator, and the two dim- 8 operators, $\mathscr{O}_{S, 0}$ and $\mathscr{O}_{S, 1}$ (cf. eqs. (2.2)(2.4)). All of these operators could arise easily in popular scenarios of new physics beyond the SM (BSM) like Composite Higgs, Little Higgs or Extra Dimensions. The LHC experiments are studying all three of them to gain sensitivity in various channels like dibosons, tribosons, precision 

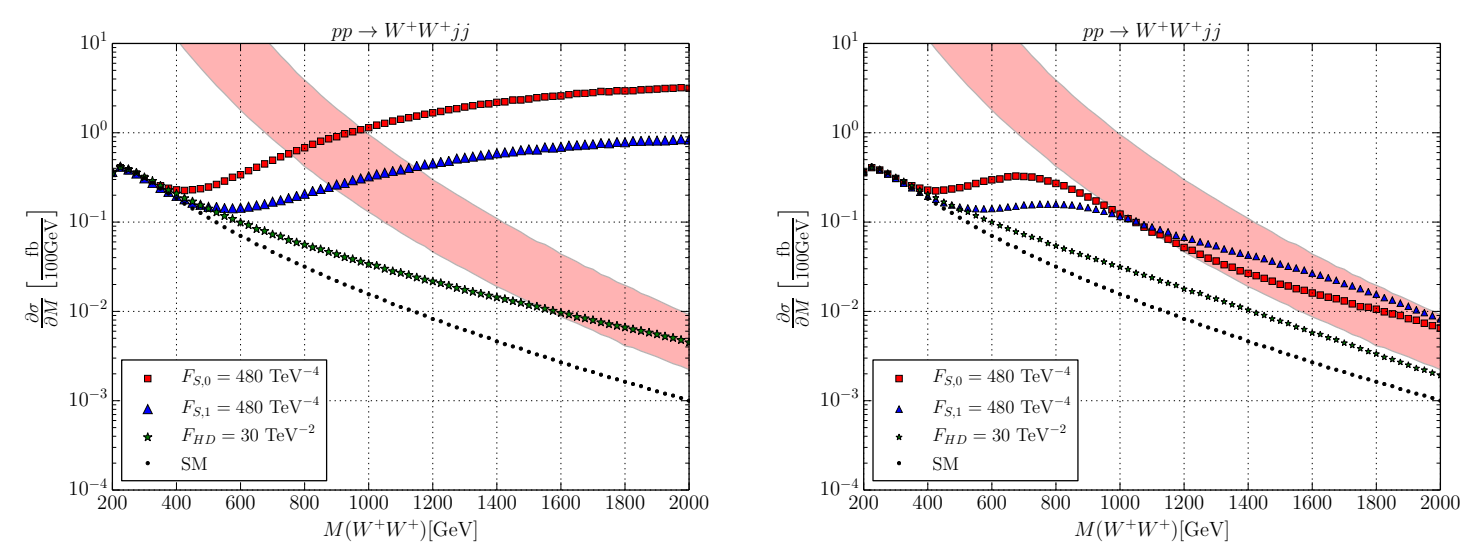

Figure 1: $p p \rightarrow W^{+} W^{+} j j$, left: naive EFT results that violate unitarity, QCD contributions neglected. The band describes maximal allowed values, due to unitarity constraints, for the differential cross section. The lower bound describes the saturation of isospin partial wave $\mathscr{A}_{20}$ and the upper bound describes the simultaneous saturation of $\mathscr{A}_{20}$ and $\mathscr{A}_{22}$, right : unitarized result. Cuts: $M_{j j}>500 \mathrm{GeV} ; \Delta \eta_{j j}>2.4 ; p_{T}^{j}>20 \mathrm{GeV} ;\left|\eta_{j}\right|>4.5$.

Higgs data and VBS. The operators are given by

$$
\begin{aligned}
& \mathscr{L}_{H D}=F_{H D} \operatorname{tr}\left[\mathbf{H}^{\dagger} \mathbf{H}-\frac{v^{2}}{4}\right] \cdot \operatorname{tr}\left[\left(\mathbf{D}_{\mu} \mathbf{H}\right)^{\dagger}\left(\mathbf{D}^{\mu} \mathbf{H}\right)\right], \\
& \mathscr{L}_{S, 0}=F_{S, 0} \operatorname{tr}\left[\left(\mathbf{D}_{\mu} \mathbf{H}\right)^{\dagger} \mathbf{D}_{v} \mathbf{H}\right] \cdot \operatorname{tr}\left[\left(\mathbf{D}^{\mu} \mathbf{H}\right)^{\dagger} \mathbf{D}^{v} \mathbf{H}\right], \\
& \mathscr{L}_{S, 1}=F_{S, 1} \operatorname{tr}\left[\left(\mathbf{D}_{\mu} \mathbf{H}\right)^{\dagger} \mathbf{D}^{\mu} \mathbf{H}\right] \cdot \operatorname{tr}\left[\left(\mathbf{D}_{v} \mathbf{H}\right)^{\dagger} \mathbf{D}^{v} \mathbf{H}\right] .
\end{aligned}
$$

Due to the unknown microscopic picture of the underlying energy giving rise to these operators, the validity range of the EFT is also a priori unknown. In this case, the unitarity condition is used to determine the validity of the EFT.

In the left-hand side of Fig. 1, the cross section for the complete LHC process $p p \rightarrow W^{+} W^{+} j j$ at leading order - computed using the Monte-Carlo generator WHIZARD $[18,19]$ with CTEQ6L PDF sets - is shown. The SM curve is compared to three curves for models which contain a single nonzero coefficient for the three different effective higher-dimensional operators, respectively. For an indication of the unitarity limits, we have included a quartic Goldstone interaction amplitude with a constant coefficient $a_{I J}=\mathrm{i}$ in the $I=2$ and $J=0,2$ isospin and spin channels and recomputed the process with this modification. The Goldstone boson scattering amplitudes are very good approximations to the scattering of longitudinal EW gauge bosons by means of the Goldstone boson equivalence theorem. By projecting the partial waves into their spin and isospin components, the optical theorem is used to determine the condition for perturbative unitarity in the same way as in [13]. Further details are listed in [7]. At high invariant mass $M_{V V}$ of the $W W$-scattering system, the enhancement the crossection by $\frac{M_{W W}^{8}}{m_{H}^{8}}$ in comparison to the SM due to the dimension eight operators are dominant. The coefficients of the higher-dimensional operators are chosen within current LHC bounds. We concentrated to the like-sign $W W$ scattering as this is the cleanest channel at the LHC with the smallest backgrounds. It only appears in the isospin two channel. In the light 


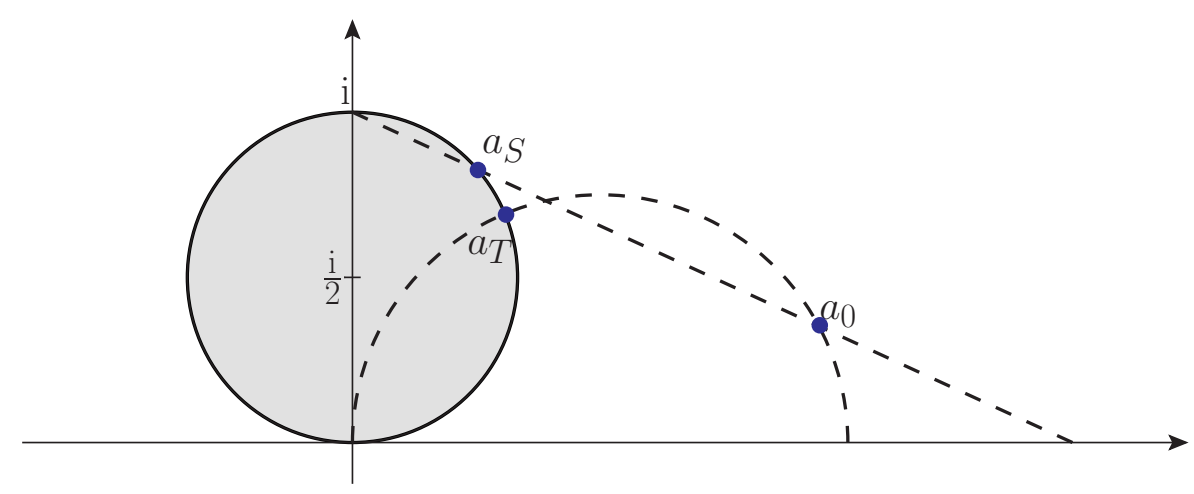

Figure 2: Geometrical representation: stereographic projection vs Thales projection.

red band, we plotted the unitarity limit by demanding that the isospin partial waves $\mathscr{A}_{20}$ and $\mathscr{A}_{22}$ for isospin two and spin zero and two, respectively, are saturated, i.e. reaching their maximally allowed value of $32 \pi$.

The predictions of the dimension eight operators violate the unitarity limit and become unphysical in an energy regime, which can be tested at the LHC. Naively, one could introduce a cut-off to forbid these unphysical events manually (a prescription also partially used by ATLAS and CMS, known as 'event clipping'). Such a cutoff could also be motivated theoretically by the argument that these events could have never arisen in a UV-complete theory. However, this leads to a sharp edge in the distribution (at level of the vector bosons) which does not resemble any sensible approximation to a UV-complete theory, and furthermore there are also experimental restrictions for doing so: In case of the $W^{+} W^{+}$scattering, the final state includes two neutrinos and the $W W$ invariant mass cannot be experimentally reconstructed. Other methods to treat this high-energy regime are by means of so-called form factors which, however, depend on at least two parameters, the exponent of the momentum dependence in the denominator (the 'multipole' parameter) and the cutoff scale which a priori has nothing to do with the scale $\Lambda$ appearing in front of the Wilson coefficients.

In order to have a meaningful description that does not depend on any additional parameters, we introduce the $T$-matrix unitarization scheme (cf. Fig. 2 in terms of the Argand circle, as described below) as a general extension of the $K$-matrix unitarization to provide event samples, which satisfy the unitarity bound. The T-matrix scheme is applicable for cases where the amplitude has an imaginary part itself already, and is also defined without relying on a perturbative expansion. For more details cf. [7]. The right-hand side of Fig. 1 shows the damping of the cross sections for high energies due to the saturation of the amplitudes.

In the first measurement of anomalous quartic gauge couplings in vector boson scattering by ATLAS [3], the non-linear representation as defined in [6] is used to probe anomalous quartic gauge couplings $\alpha_{4}$ and $\alpha_{5}$,

$$
\begin{aligned}
& \mathscr{L}_{\alpha_{4}}=\alpha_{4} \operatorname{tr}\left[\mathbf{V}_{\mu} \mathbf{V}_{v}\right] \cdot \operatorname{tr}\left[\mathbf{V}^{\mu} \mathbf{V}^{v}\right], \\
& \mathscr{L}_{\alpha_{5}}=\alpha_{5} \operatorname{tr}\left[\mathbf{V}_{\mu} \mathbf{V}^{\mu}\right] \cdot \operatorname{tr}\left[\mathbf{V}_{v} \mathbf{V}^{v}\right] .
\end{aligned}
$$


$\mathscr{L}_{\alpha_{4}}$ and $\mathscr{L}_{\alpha_{5}}$ include the subset of couplings of $\mathscr{L}_{S, 0}$ and $\mathscr{L}_{S, 1}$ excluding all anomalous couplings with at least one Higgs. However, anomalous couplings involving the Higgs can be neglected in a study of vector boson scattering at the LHC due to the small Yukawa interaction of incoming quarks and specialized triggers for outgoing vector bosons. Therefore, both parametrization are equivalent to study anomalous quartic gauge couplings in vector boson scattering processes. Limits on the anomalous couplings $\alpha_{4}$ and $\alpha_{5}$ can be directly translated to limits of $F_{S, 0}$ and $F_{S, 1}$, with or without K/T-matrix unitarization, via

$$
\begin{aligned}
& F_{S, 0}=16 \frac{\alpha_{4}}{v^{4}} \\
& F_{S, 1}=16 \frac{\alpha_{5}}{v^{4}}
\end{aligned}
$$

For example, a conversion of the observed one-dimensional 95\% interval in [3] leads with eq. (2.7) to following observed $95 \%$ interval for $F_{S, 0}$ and $F_{S, 1}$ :

$$
\begin{aligned}
& -590 \mathrm{TeV}^{-4}<F_{S, 0}<680 \mathrm{TeV}^{-4} \text { for } \quad F_{S, 1}=0, \\
& -420 \mathrm{TeV}^{-4}<F_{S, 1}<510 \mathrm{TeV}^{-4} \text { for } \quad F_{S, 0}=0 .
\end{aligned}
$$

The T-matrix scheme is only one possible extrapolation for high-energy scenarios. All physical scenarios have to fullfil the unitarity condition which is graphically represented by the Argand circle. If no new physics is involved in the electroweak sector, the elastic scattering amplitude of the Standard model will stay at the origin on the bottom of the Argand circle (Fig. 3a). If the EFT is naively added, amplitudes start to rise and will leave the Argand circle to finally violate unitarity (cf. Fig. 3b, as there are no new degrees of freedom in the strict EFT, the amplitude can never develop an imaginary part to return to the Argand circle). To remedy this unphysical behavior of the amplitude, unitarization prescriptions are introduced to project the amplitude back onto the Argand circle. T-matrix unitarization saturizes the amplitude, in the sense that it is equivalent to an infinitely broad resonance at infinity, similarly to a strongly interacting continuum present over an extended range in momentum space. Practically, it will project the corresponding isospin-spin amplitude to its maximally allowed absolute value at high energies. Another option to correct the unphysical EFT prediction is using the form-factor scheme, a possible case of entering the inelastic regime with additional channels opening up (cf. Fig. 3d). A third approach would be the addition of explicit resonances (either weakly or strongly coupled), which could be (part of the) origin for the dim- 8 operators (cf. Fig. 3e). Here, the amplitude will ideally fall again beyond the resonance, but could show a rise again due to continuum contributions or the onset of a further resonance.

\section{Resonances and Simplified Models}

As the LHC is intended to be a discovery machine, it might be advantageous to assume that a new resonance or particle might be within the kinematic reach of the machine, especially given the high amount of luminosity to be collected in runs II and III. In order to be as general as possible in studying what kind of resonances could show up in vector boson scattering - specific models would be Two-Higgs double models, including the (N)MSSM, Composite Higgs, Little Higgs (for limits cf. e.g. [14]), Twin Higgs, etc. - we classify all resonances that can couple to the electroweak 


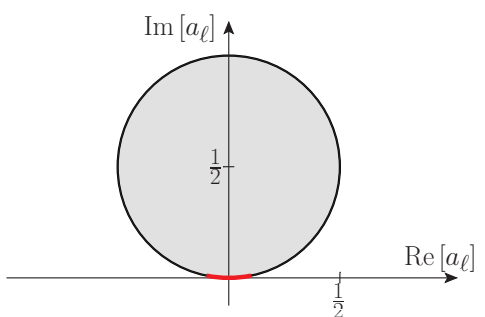

(a) SM

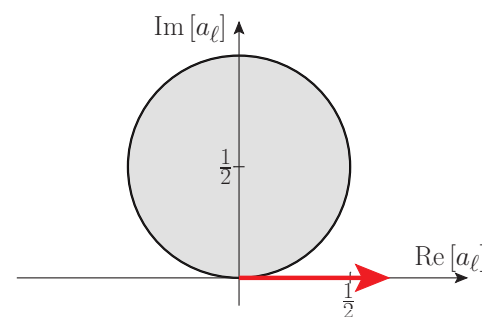

(b) Bare EFT, high-energy

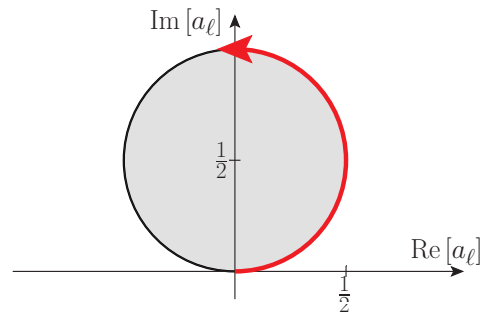

(c) Saturation

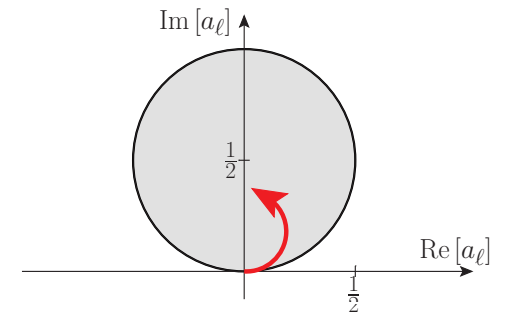

(d) Inelastic channels

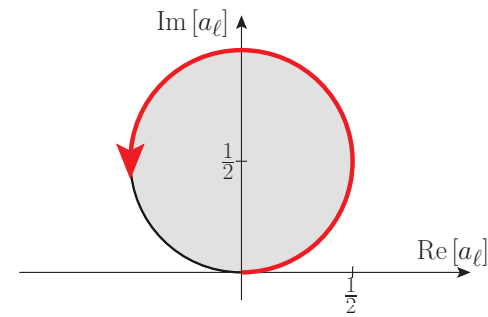

(e) Resonance

Figure 3: Possible scenarios for scattering amplitudes respecting the Argand circle.

diboson systems according to their spin and isospin quantum numbers. For simplicity, we neglect couplings to photons, but of course they are present due to EW gauge invariance. These possible resonances can be categorized in terms of the approximate $S U(2)_{L} \times S U(2)_{R}$ symmetry, which is a good approximation for weak boson scattering, and the spin. The $(0,0)$ and the $(1,1)$ representations of the $S U(2)_{L} \times S U(2)_{R}$ are abbreviated as isoscalar and isotensor, respectively. We can distinguish the resonances for elastic vector boson scattering into an isoscalar scalar $\sigma$, an isoscalar tensor $f$, an isotensor scalar $\phi$ and an isotensor tensor $X$. The interaction with longitudinal vector bosons is modeled by the following currents:

$$
\begin{aligned}
J_{\sigma}=F_{\sigma} \operatorname{tr}\left[\left(\mathbf{D}_{\mu} \mathbf{H}\right)^{\dagger} \mathbf{D}^{\mu} \mathbf{H}\right], \\
J_{\phi}=F_{\phi}\left(\left(\mathbf{D}_{\mu} \mathbf{H}\right)^{\dagger} \otimes \mathbf{D}^{\mu} \mathbf{H}+\frac{1}{8} \operatorname{tr}\left[\left(\mathbf{D}_{\mu} \mathbf{H}\right)^{\dagger} \mathbf{D}^{\mu} \mathbf{H}\right]\right) \tau^{a a}, \\
J_{f}^{\mu v}=F_{f}\left(\operatorname{tr}\left[\left(\mathbf{D}^{\mu} \mathbf{H}\right)^{\dagger} \mathbf{D}^{v} \mathbf{H}\right]-\frac{c_{f}}{4} g^{\mu v} \operatorname{tr}\left[\left(\mathbf{D}_{\rho} \mathbf{H}\right)^{\dagger} \mathbf{D}^{\rho} \mathbf{H}\right]\right), \\
J_{X}^{\mu v}=F_{X}\left[\frac{1}{2}\left(\left(\mathbf{D}^{\mu} \mathbf{H}\right)^{\dagger} \otimes \mathbf{D}^{v} \mathbf{H}+\left(\mathbf{D}^{v} \mathbf{H}\right)^{\dagger} \otimes \mathbf{D}^{\mu} \mathbf{H}\right)-\frac{c_{X}}{4} g^{\mu v}\left(\mathbf{D}_{\rho} \mathbf{H}\right)^{\dagger} \otimes \mathbf{D}^{\rho} \mathbf{H}\right. \\
\left.+\frac{1}{8}\left(\operatorname{tr}\left[\left(\mathbf{D}^{\mu} \mathbf{H}\right)^{\dagger} \mathbf{D}^{v} \mathbf{H}\right]-\frac{c_{X}}{4} g^{\mu v} \operatorname{tr}\left[\left(\mathbf{D}_{\rho} \mathbf{H}\right)^{\dagger} \mathbf{D}^{\rho} \mathbf{H}\right]\right)\right] \tau^{a a} .
\end{aligned}
$$

Here, $\mathbf{H}=\frac{1}{2}\left(\mathbb{1}(v+H)-i w^{a} \tau^{a}\right)$, and $\tau^{a a}$ is the tensor-product representation for the isotensor case. With those resonances at hand, parameterized simply by their masses and widths, together with the currents above, one can integrate them out again and derive the corresponding Wilson coefficients of the dim-8 operators $\mathscr{O}_{S, 0}$ and $\mathscr{O}_{S, 1}$ in the section before, for all cases considered above. The coefficients are listed in table 1 . 

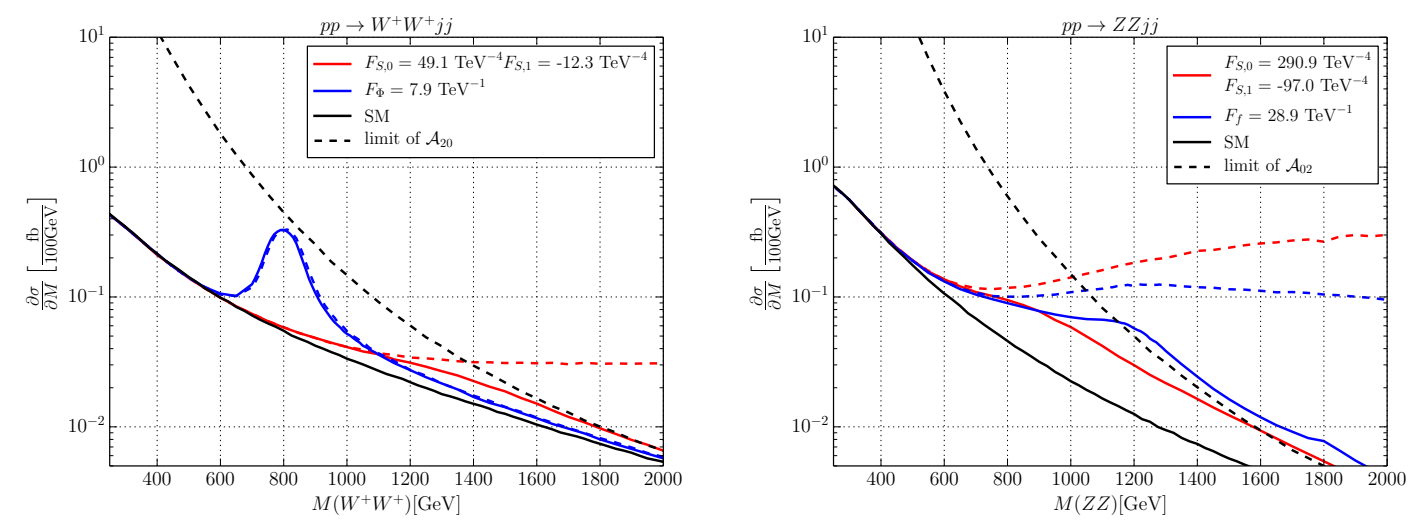

Figure 4: Differential cross sections of a scalar-isotensor resonance (left) and an isoscalar-tensor resonance (right). Solid line: unitarized results, dashed lines: naive result, black dashed line: limit of saturation of $\mathscr{A}_{22}\left(W^{+} W^{+}\right) / \mathscr{A}_{02}(Z Z)$. Cuts: $M_{j j}>500 \mathrm{GeV} ; \Delta \eta_{j j}>2.4 ; p_{T}^{j}>20 \mathrm{GeV}$; $\left|\eta_{j}\right|>4$.5. Left: $p p \rightarrow W^{+} W^{+} j j$, scalar-isotensor with $m_{\phi}=800 \mathrm{GeV}$ and $\Gamma_{\phi}=80 \mathrm{GeV}$, right: $p p \rightarrow Z Z j j$, strongly interacting isotensor scalar with $m_{f}=1200 \mathrm{GeV}$ and $\Gamma_{f}=480 \mathrm{GeV}$.

\begin{tabular}{c|cccc} 
& $\sigma$ & $\phi$ & $f$ & $X$ \\
\hline$F_{S, 0}$ & - & 2 & 15 & 5 \\
$F_{S, 1}$ & $1 / 2$ & $-1 / 2$ & -5 & -35
\end{tabular}

Table 1: Relation of resonance width $\Gamma$ and mass $m$ to the corresponding dimension eight operator coefficients in the low-energy effective field theory. The factors listed in the table have to be multiplied by $32 \pi \Gamma / m^{5}$.

Tensor resonances as they could arise as Kaluza-Klein recurrences of a higher-dimensional gravity theory, but also as analogues to tensor mesons in a composite model, are particularly interesting. They usually give the largest signal contributions, as here the maximum number of spin components are involved in the scattering, namely five, compared to scalar and vector cases. There is a substantial difference in the theoretical treatment of those intrinsic spin degrees of freedom when dealing with the tensor resonance on-shell and off-shell. In a full Monte-Carlo simulation (cf. below), one actually simulates the final state and always has the tensor resonance in offshell configurations. Using the analogue of unitarity gauge for tensors, the propagators lead to a bad high-energy behavior of the amplitudes. A symmetric tensor field $f_{\mu \nu}$ has 10 components which are reduced by the on-shell conditions to five physical components within the Fierz-Pauli Lagrangian [15]. These conditions are the tracelessness, $f_{\mu}{ }^{\mu}=0$ and the transversality, $\partial_{\mu} f^{\mu v}=0$. However, these Fierz-Pauli conditions are not valid off-shell, so we use the Stückelberg mechanism $[16,17]$ to make the off-shell high-energy behavior explicit. Onshell, there is only the tensor field, $f^{\mu v}$, while off-shell there is a vector field, $A^{\mu} \sim \partial_{v} f^{\mu v}$, which corresponds to the transversality condition, a scalar implementing the fully contracted transversality, $\phi \sim \partial_{\mu} \partial_{v} f^{\mu v}$, and another scalar corresponding to the tracelessness, $\sigma \sim f_{\mu}{ }^{\mu}$. By gauge fixing, one of the scalar degrees 

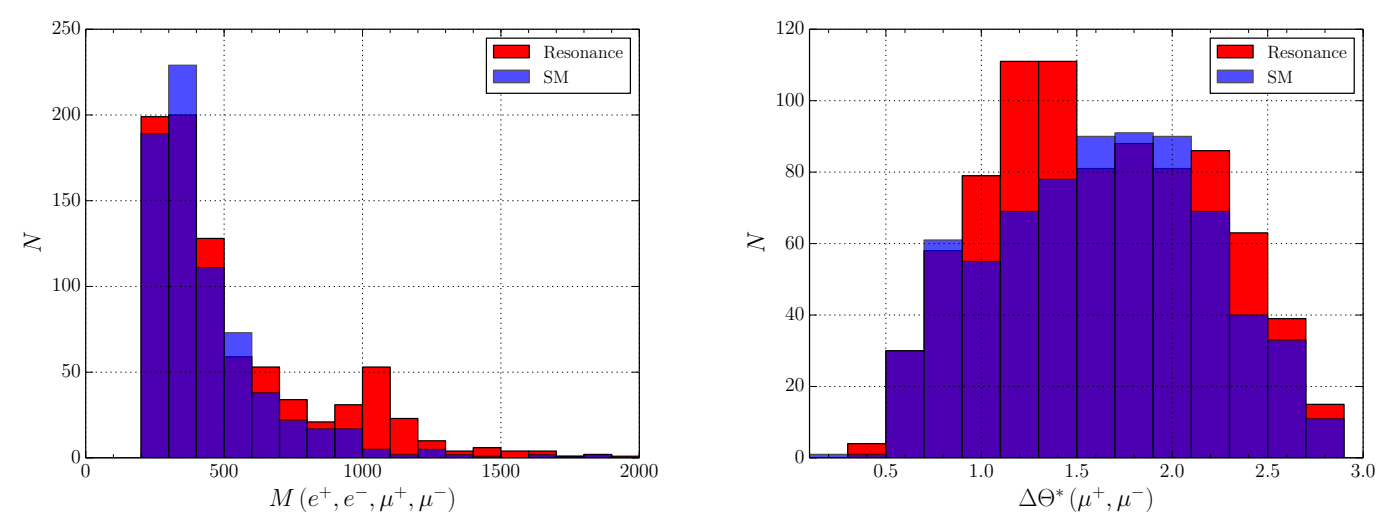

Figure 5: Isoscalar-tensor resonance at $m_{f}=1000 \mathrm{GeV}$ and $\Gamma_{f}=100 \mathrm{GeV} p p \rightarrow e^{+} e^{-} \mu^{+} \mu^{-} j j$ at $\sqrt{s}=14 \mathrm{TeV}$ with luminosity of $3000 \mathrm{fb}^{-1}$, with cuts $M_{j j}>500 \mathrm{GeV} ; \Delta \eta_{j j}>2.4 ; p_{T}^{j}>20 \mathrm{GeV}$; $\left|\eta_{j}\right|>4.5 ; 100 \mathrm{GeV}>M_{e^{+} e^{-}}>80 \mathrm{GeV} ; 100 \mathrm{GeV}>M_{\mu^{+} \mu^{-}}>80 \mathrm{GeV}$.

of freedom is redundant: $\sigma=-\phi$. The technical details together with the full Lagrangians and currents for the Fierz-Pauli as well as the Stïckelberg picture can be found in [7].

Fig. 4 shows two examples how differential invariant mass distributions of the diboson system behave at the LHC in the presence of such resonances. Both plots show different resonances in different scenarios: the left plot a narrow isotensor scalar with mass $m_{\phi}=800 \mathrm{GeV}$ and width $\Gamma_{f}=80 \mathrm{GeV}$, the right one a strongly-interacting scenario with a broad isoscalar-tensor resonance of mass $m_{f}=1.2 \mathrm{TeV}$ and width $\Gamma_{f}=480 \mathrm{GeV}$. The left plot shows the like-sign $W^{+} W^{+}$channel, the right one the opposite-sign $W^{+} W^{-} \rightarrow Z Z$ channel, respectively. General cuts for selection and signal/background enhancement are shown in the caption. The full black line is the SM, the black dashed line shows the corresponding unitarity limit of the leading partial wave amplitude, the full blue line shows the SM with the corresponding resonance, while full red line depicts the approximation with the two Wilson coefficients, $F_{S, 0}$ and $F_{S, 1}$. Clearly, if explicit resonances are in the kinematic reach of the LHC, the EFT is no longer a viable approximation in any case. Note that even in the simulation with an explicit resonance, T-matrix unitarization has been applied to unitarize the high-energy tail of the distribution. As here the amplitudes do have explicit complex poles, T-matrix unitarization is actually needed.

We have implemented the complete set of longitudinal dimension- 6 and dimension- 8 operators together with the prescription of K-/T-matrix unitarization (for longitudinal VBS) in the Monte Carlo event generator WHIZARD $[18,19]$. It contains a quite elaborate machinery for QCD precision physics, where it uses the color flow formalism [20], it has its own parton shower implementations [21], and quite recently has successfully demonstrated its QCD NLO capabilities [22]. WHIZARD has been used for a plethora of BSM studies, and is able to read in external models, e.g. via [23]. Using this implementation, we simulated vector boson scattering at the LHC with its design energy of $\sqrt{s}=14 \mathrm{TeV}$ for all kinds of narrow and wide resonances of different spin and isospin. Fig. 5 shows an example of an isoscalar tensor resonance of mass $m_{f}=1 \mathrm{TeV}$ and a width of $\Gamma_{f}=100 \mathrm{GeV}$ in the scattering of opposite-sign $W$ s into two Zs. A standard set of selection cuts are mentioned in the caption of the figure. The left plot shows the invariant mass of the diboson 
system, which in this case is fully reconstructible, while the right plot shows the distribution of the opening angle of the two muons from one of the Zs. The latter is one of the angular observables that could be used to discriminate the spin of such resonances. More examples can be found in [7].

\section{Conclusions}

The search for new physics in the electroweak sector in vector boson scattering at the LHC can be studied in the context of effective field theory, however, the introduction of dim- 6 and dim- 8 operators leads to a very limited range of applicability of the EFT ansatz. In many models, dim- 8 operators could be the leading contributions where tree-level effects are forbidden by symmetries, and first contributions come in at the one-loop level, i.e. at dim-8. LHC as a hadron collider probes a vast range of energy scales, and high-energy events tend to (over-)dominate the exclusion limits (or search potentials) for new models. In most cases this is due to wrong assumptions on the underlying model, if EFT-based approaches in regimes are used where perturbative unitarity is lost. We studied examples of a dim- 6 and two dim- 8 operators and derived unitarity limits for the different spin and isospin channels in the scattering of longitudinal electroweak vector bosons. Then, a unitarization method, T-matrix unitarization, that is parameter-free and that is an extension of the "classic" K-matrix unitarization has been applied to produce results that are physically meaningful. The T-matrix unitarization has certain advantages, as it is defined for amplitudes that are intrinsically complex, and does not rely on the existence of a perturbative expansion. For weakly coupled amplitudes without imaginary parts it is identical to K-matrix unitarization. This procedure is not just an academic exercise, it allows to produce Monte Carlo events that could actually come from a quantum field theory realized in nature. Furthermore, it is itself a possible limit of a a strongly interacting continuum like in QCD or close to a quasi-conformal fixed point, or it could correspond to a strongly interacting model right below the onset of a new resonance that is just a little bit outside the kinematical reach of LHC. Even if the T-matrix prescription is not the correct extrapolation of the EFT, it can be interpreted as upper bound of the corresponding elastic channels, e.g. isospin-spin channels, due to its saturizing character. In combination with its property, that it leaves an interaction matrix invariant, which already satisfies unitarity, it can always used as "fail-safe"-mechanism to generate event samples, which respect fundamental physical properties. We show examples of cross sections as well as kinematic and angular distributions to show the effects between "bare" EFT and unitarized simulations.

Beyond this parameter-less approach to new physics in vector boson scattering, we provided a set of simplified models taking the SM added by all possible resonances in the spin-isospin channels to which two EW vector bosons can couple. We focused on scalar and tensor resonances, while vector resonances are more complicated due to their potential mixing with the EW bosons. To account for effects of particularly strongly interacting models, in addition higher-dimensional operators can be added. Also, adding just single resonances does not lead to renormalizable models with sound high-energy behavior, hence, we also applied T-matrix unitarization to the simplified models. In order to start with a prescription that already has the best possible high-energy behavior, we isolated the scalar and vector degrees of freedom in massive tensor fields via the Stückelberg mechanism to represent explicitly the bad behavior of tensor propagators in unitarity gauge. We 
concluded with a fully differential example for a simplified model with an isoscalar tensor resonance.

An updated study for vector boson scattering in future lepton colliders [24, 25] is completed in [26]. Further work will be devoted to the study of transverse $W$ and $Z$ polarizations, the discussion of vector resonances as well a implementation of the T-matrix for $2 \rightarrow 3$ processes, which is relevant for triple weak boson production.

\section{Acknowledgements}

MS thanks the organizers for a very enjoyable and interesting conference.

\section{References}

[1] G. Aad et al. [ATLAS Collaboration], Phys. Lett. B 716, 1 (2012) doi:10.1016/j.physletb.2012.08.020 [arXiv:1207.7214 [hep-ex]].

[2] S. Chatrchyan et al. [CMS Collaboration], Phys. Lett. B 716, 30 (2012) doi:10.1016/j.physletb.2012.08.021 [arXiv:1207.7235 [hep-ex]].

[3] G. Aad et al. [ATLAS Collaboration], Phys. Rev. Lett. 113, no. 14, 141803 (2014) [arXiv:1405.6241 [hep-ex]].

[4] The ATLAS collaboration [ATLAS Collaboration], ATLAS-CONF-2014-013.

[5] CMS Collaboration [CMS Collaboration], CMS-PAS-FSQ-13-008.

[6] A. Alboteanu, W. Kilian and J. Reuter, JHEP 0811, 010 (2008) [arXiv:0806.4145 [hep-ph]].

[7] W. Kilian, T. Ohl, J. Reuter and M. Sekulla, Phys. Rev. D 91 (2015) 096007 [arXiv:1408.6207 [hep-ph]].

[8] W. Kilian, T. Ohl, J. Reuter and M. Sekulla, Phys. Rev. D 93, no. 3, 036004 (2016) doi:10.1103/PhysRevD.93.036004 [arXiv:1511.00022 [hep-ph]].

[9] M. Sekulla Ph.D. thesis, University of Siegen, 2015

[10] J. Reuter, W. Kilian, T. Ohl and M. Sekulla, arXiv:1605.09594 [hep-ph].

[11] M. Baak et al., arXiv:1310.6708 [hep-ph].

[12] C. Degrande et al., arXiv:1309.7890 [hep-ph].

[13] B. W. Lee, C. Quigg and H. B. Thacker, Phys. Rev. Lett. 38, 883 (1977); Phys. Rev. D 16, 1519 (1977).

[14] J. Reuter and M. Tonini, JHEP 1302, 077 (2013) [arXiv:1212.5930 [hep-ph]]. J. Reuter, M. Tonini and M. de Vries, arXiv:1307.5010 [hep-ph]; JHEP 1402, 053 (2014) [arXiv:1310.2918 [hep-ph]].

[15] M. Fierz and W. Pauli, Proc. Roy. Soc. Lond. A 173 (1939) 211.

[16] E. C. G. Stueckelberg, Helv. Phys. Acta 11, 225 (1938).

[17] E. C. G. Stueckelberg, Helv. Phys. Acta 15 (1942) 23.

[18] W. Kilian, T. Ohl and J. Reuter, WHIZARD: Simulating Multi-Particle Processes at LHC and ILC, Eur. Phys. J. C 71, 1742 (2011) [arXiv:0708.4233 [hep-ph]]. 
[19] M. Moretti, T. Ohl and J. Reuter, O’Mega: An Optimizing matrix element generator, In *2nd ECFA/DESY Study 1998-2001* 1981-2009 [hep-ph/0102195].

[20] W. Kilian, T. Ohl, J. Reuter and C. Speckner, JHEP 1210, 022 (2012) [arXiv:1206.3700 [hep-ph]].

[21] W. Kilian, J. Reuter, S. Schmidt and D. Wiesler, JHEP 1204, 013 (2012) [arXiv:1112.1039 [hep-ph]].

[22] B. Chokoufe Nejad, W. Kilian, J. Lindert, S. Pozzorini, J. Reuter, C. Weiss, in preparation; B. Chokoufe Nejad, W. Kilian, J. Reuter and C. Weiss, PoS EPS -HEP2015, 317 (2015) [arXiv:1510.02739 [hep-ph]]; C. Weiss, B. Chokoufe Nejad, W. Kilian and J. Reuter, PoS EPS -HEP2015, 466 (2015) [arXiv:1510.02666 [hep-ph]]; N. Greiner, A. Guffanti, T. Reiter and J. Reuter, Phys. Rev. Lett. 107, 102002 (2011) [arXiv:1105.3624 [hep-ph]]; T. Binoth, N. Greiner, A. Guffanti, J. Reuter, J.-P. Guillet and T. Reiter, Phys. Lett. B 685, 293 (2010) [arXiv:0910.4379 [hep-ph]]; W. Kilian, J. Reuter and T. Robens, Eur. Phys. J. C 48, 389 (2006) [hep-ph/0607127].

[23] N. D. Christensen, C. Duhr, B. Fuks, J. Reuter and C. Speckner, Eur. Phys. J. C 72, 1990 (2012) [arXiv:1010.3251 [hep-ph]].

[24] H. Baer et al., arXiv:1306.6352 [hep-ph].

[25] T. Behnke et al., arXiv:1306.6329 [physics.ins-det].

[26] C. Fleper, W. Kilian, J. Reuter and M. Sekulla, arXiv:1607.03030 [hep-ph]. 